\title{
Long-Term Follow-Up After Complete Resection of Well-Differentiated Cancer Confined to the Thyroid Gland
}

\author{
Stephan A. Vorburger, MD, MCR ${ }^{1}$, Lucia Übersax, MS ${ }^{1}$, Stefan W. Schmid, MD ${ }^{1}$, Mariette Balli, MD $^{2}$, \\ Daniel Candinas, $\mathrm{MD}^{1}$, and Christian A. Seiler, MD, $\mathrm{MHA}^{1}$ \\ ${ }^{1}$ Department of Visceral and Transplantation Surgery, Inselspital, University Hospital Bern, University of Bern, Bern, \\ Switzerland; ${ }^{2}$ Institute of Pathology, University of Bern, Bern, Switzerland
}

\begin{abstract}
Background. Papillary or follicular thyroid carcinomas exhibit a relatively benign course. Hence, long-term follow-up studies with well-defined disease stages and treatment details are needed to evaluate treatment strategies.
\end{abstract}

Methods. Patients who underwent complete resection of well-differentiated thyroid carcinoma (WDTC) confined to the thyroid gland between 1972 and 1990 identified from a prospective database were assessed. Follow-up was performed by interview, review of patient charts, and analysis of the Death Registry. Primary endpoints were overall survival (OS) and disease-specific survival (DSS). Review of histology was performed and extent of thyroid resection, postoperative therapy, and recognized prognostic factors but not lymphadenectomy were evaluated.

Results. Of 2,867 patients, 213 had complete resection of WDTC confined to the thyroid gland. Follow-up was completed in 166 patients with median age 54.2 (range, 20-85) years, and median follow-up of 27.2 (range, 15.634.5 ) years. The 10- and 20-year OS was 71 and $55 \%$, respectively. DSS at 10 and 20 years was 81 and $69 \%$, respectively, and correlated with age, histology, tumor size, radio-iodide ablation (RIA), and external beam irradiation (EBR) treatment. No patient died of WDTC more than 18 years after resection. Total or near-total thyroidectomy without lymphadenectomy was not superior to partial thyroidectomy. In multivariate analysis for DSS, age was the dominant factor, which correlated with histology.

(C) Society of Surgical Oncology 2009

First Received: 31 January 2009;

Published Online: 5 August 2009

S. A. Vorburger, MD, MCR

e-mail: svorburger@bluewin.ch
Conclusion. After a median follow-up of 27 years, about one-third of patients died of WDTC. Age, histology and postoperative therapy but not extent of thyroid resection determined DSS.

Thyroid cancer accounts for roughly $0.5-1.5 \%$ of all cancers. Although it is a rare cancer, it is the most common endocrine malignancy. ${ }^{1}$ More than $94 \%$ of malignant thyroid tumors arise from follicular epithelial cells to form well-differentiated papillary or follicular carcinomas (WDTC) (reviewed in Sherman ${ }^{2}$ ). Most WDTC display a relatively benign disease course with a reported 10-year disease-specific survival (DSS) of up to $96 \%$ for papillary thyroid carcinoma (PTC) and $90 \%$ for follicular thyroid carcinoma (FTC). ${ }^{3,4}$

The low tumorigenicity of this cancer renders the determination of optimal treatment strategies through prospective clinical trials with meaningful follow-up periods nearly impossible. Therefore, an evaluation of factors that influence overall survival (OS) and DSS is dependent on retrospective studies based either on large study populations or on longterm follow-up. Both approaches have inherent limitations. Most large observational studies with median follow-up of 10 and more years are based on databases from cancer registries not specific for thyroid tumors. These are limited by the lack of detailed descriptions of tumor characteristics and/ or exact treatments applied. ${ }^{4,5}$ On the other hand, and with the exception of the studies from the Mayo Clinics, longterm follow-up studies from well-defined study groups that are typically derived from single-institutional thyroid-specific prospective databases are hampered by small sample sizes and treatment inconsistencies that occur during the long time over which patients were included. ${ }^{6,7}$

Various clinicopathological prognostic factors have been integrated in more than 15 different tumor staging 
systems for WDTC. ${ }^{8}$ Most systems are based on the risk factors age, tumor size, and presence of metastatic disease used to stratify patients in risk groups for disease-specific survival (DSS). The MACIS-, EORTC-, and AJCC/UICC TNM staging systems reportedly have the best predictive accuracy (AJCC/UICC 6th edition). ${ }^{9}$ Of these staging instruments, only the MACIS system integrates completeness of resection. ${ }^{10}$ Additional factors associated with disease recurrence but not necessarily DSS were the presence of extrathyroidal spread, regional lymph node metastases, and postoperative radio-iodine ablation therapy (RIA). ${ }^{11-14}$

As for most solid tumors, surgical resection is the mainstay of treatment. Whereas the importance of complete tumor resection is acknowledged, the extent of additional thyroid tissue that must be removed and the implementation of lymphadenectomy remain controversial. Proponents of total thyroidectomy justify this approach because of the high rate of multifocality with bilateral disease in PTC. In addition, total thyroidectomy facilitates the detection of postoperative recurrence and metastasis by radioiodine uptake and thyroglobulin serum level measurements. Indeed, many investigators found that more extensive resection of DTC with total or near-total thyroidectomy results in a lower incidence of local recurrences, although it does not influence DSS. ${ }^{15-21}$ Recently, Bilimoira et al. ${ }^{22}$ showed a survival benefit after total thyroidectomy over lobectomy in PTC larger than $1 \mathrm{~cm}$ analyzing a cancer registry database. Proponents of limited resection base their strategy on publications reporting that postsurgical complications increased with more extensive resection. Data about DSS in these studies seem to justify limited thyroid resection, favoring lobectomy of the affected side over total thyroidectomy. ${ }^{7,14,23-32}$

In this retrospective study of a well-defined patient group with WDTC confined to the thyroid gland undergoing complete resection of the tumor, we sought to define long-term outcomes, to evaluate previously described prognostic factors for DSS in WDTC, and to determine the influence of the extent of thyroid resection, in particular.

\section{PATIENTS AND METHODS}

Since 1972, clinical and pathologic data, treatment rendered, intraoperative findings, and follow-up information for all patients undergoing thyroid surgery at the Department of Visceral and Transplantation Surgery, University Hospital of Bern, have been entered in a comprehensive databank. From the group of patients with WDTC, papillary (PTC), or follicular (FTC) carcinomas who were surgically treated between 1972 and 1990, we identified patients who had complete resection of primary tumors confined to the thyroid gland, with no macroscopic spread to adjacent lymph nodes. To avoid misinterpretation for inconsistencies in lymph node surgery, patients who had enlarged lymph nodes and, hence, lymphadenectomy were excluded. Patients who died within 30 days of initial thyroid resection were excluded. Distant metastasis was determined by whole body scan at the beginning of RIA treatment, and positive patients were excluded. Histology was confirmed or restaged by an actual review of tumor blocks at the Pathology Institute of the University of Bern. These patients formed the study population.

The following definitions were used. The time of diagnosis was defined as the date of initial resection. Tumor size was defined as the maximum dimension of the resected tumor described in the pathologic report. The maximal size of a T1 tumor was $2 \mathrm{~cm}$, of a T2 tumor was $>2$ to $\leq 4 \mathrm{~cm}$, and of a T3 tumor was $>4 \mathrm{~cm}$, with the entire tumor limited to the thyroid. The TNM tumor stage was determined according to the UICC AJCC TNM classification of malignant tumors ${ }^{33}$ : In the absence of distant metastasis, age $<45$ years or tumor size $\mathrm{T} 1$ in older patients defines Stage I. Tumor size T2 in patients older than 45 years is classified as Stage II; Stage III correlates to a T3 tumor or any lymph node involvement in patients $>45$ years. For PTC, the staging system from the Mayo clinic based on metastasis, age, completeness of resection, invasion, and tumor size (MACIS), was also used. ${ }^{10}$ The definition of complete surgical excision was based on information from the surgical and histopathologic reports indicating complete removal of the entire primary tumor. The extent of resection was defined as total thyroid resection (TR) if less than $1 \mathrm{~g}$ (near-total) of thyroid tissue was left behind (partial resection (PR) if more than $1 \mathrm{~g}$ was left in place).

Postoperative radio-iodide ablation consisted of postoperative application of $30-200 \mathrm{mCi}{ }^{131} \mathrm{I}$, and a posttherapy scan was routinely performed. External beam irradiation (EBR) consisted of a postoperative application of $30 \mathrm{~Gy}$, typically.

A retrospective analysis of the data, including a review of patient charts, was performed in 2007. The follow-up was achieved by telephone-interview, review of patient charts from family practitioners, and analysis of the Swiss Death Registry for cause of death in nonsurvivors. Primary endpoints were overall survival (OS) and DSS. Due to the long follow-up period, disease-free survival could not be reliably determined.

For statistical analysis, associations among categorical variables were tested using the chi-square or Fisher exact test, where appropriate. The OS and DSS were defined as the time from initial resection to the date of death from any cause (OS), or death from disease (DSS). Patients who did not die were censored for OS or DSS analysis at the time of last follow-up. The OS and DSS curves were generated 
using the Kaplan-Meier method. ${ }^{34}$ The log-rank test was used to test for differences among patient groups. Independent prognostic factors were examined using the Cox proportional hazards regression model. ${ }^{35}$ Statistical analyses were performed using NCSS software (NCSS and PASS. Number Cruncher Statistical Systems. and Power Analysis Statistical Software; Kaysville, UT). A $P$ value of $<.05$ was considered significant.

\section{RESULTS}

A total of 213 Swiss patients with primary WDTC were treated at the University Hospital of Bern during the study period (1972-1990). Of these, 186 had tumors confined to the thyroid (no extension beyond the thyroid capsule) that were resected with tumor-free margins. Patients who had lymphadenectomy caused by enlarged lymph nodes were excluded. This led to a highly selected group of 186 patients with macroscopically localized disease and microscopically confirmed complete resection of the carcinoma (Table 1). A review of histology led to the exclusion of 5 patients (4 adenomas, 1 anaplastic carcinoma) and restaging of 14 initial FTCs to PTC. Follow-up was complete in 166 of the 181 patients. The median follow-up time was 27.2 years (range, 15.6-34.3). The distribution of clinicopathologic factors in all 166 patients with complete resection of localized WDTC is summarized in Table 1. The median age of the study population at

TABLE 1 Characteristics and treatment of 166 patients with well-differentiated thyroid cancer

\begin{tabular}{|c|c|c|c|}
\hline \multirow[t]{2}{*}{ Characteristics } & \multicolumn{3}{|l|}{ No. of patients } \\
\hline & \multicolumn{3}{|l|}{ All } \\
\hline Thyroid surgery & \multicolumn{3}{|l|}{2867} \\
\hline Primary well-differentiated thyroid carcinoma & \multicolumn{3}{|l|}{213} \\
\hline Localized disease + resection margin free & \multicolumn{3}{|l|}{186} \\
\hline & Total & PTC & FTC \\
\hline Questionnaire and follow-up completed & 181 & $69(42 \%)$ & $102(56 \%)$ \\
\hline Before revision & & & Huertle $15(9 \%)$ \\
\hline Histologic revision & 166 & $83(50 \%)$ & $\begin{array}{l}83(50 \%) \text { (including } \\
\quad \text { Huertle, } n=15[9 \%] \text { ) }\end{array}$ \\
\hline Median f/u (range), years & $27.2(15.8-34.3)$ & $26.3(15.8-34.3)$ & $29.8(16.7-33.9)$ \\
\hline Median age (range), years & $54.2(20.2-85)$ & $42.2(20.2-83.2)$ & $63.5(20.2-85)$ \\
\hline Age $<60$ years & $95(57 \%)$ & $61(64 \%)$ & $34(36 \%)$ \\
\hline Men/women & $51 / 115$ & $27 / 56$ & $24 / 59$ \\
\hline \multicolumn{4}{|l|}{ Tumor size T (UICC TNM 6th ed.) } \\
\hline T1 $(<2 \mathrm{~cm}) ;[$ pT1a $(<1 \mathrm{~cm})]$ & & $42(50 \%) ;[26(50 \%)]$ & $8(9 \%) ;[0]$ \\
\hline $\mathrm{T} 2$ & & $18(22 \%)$ & $13(16 \%)$ \\
\hline \multirow[t]{2}{*}{$\mathrm{T} 3(>4 \mathrm{~cm})$} & & $23(28 \%)$ & $62(75 \%)$ \\
\hline & $n(\%)$ & $n(\%)$ & $n(\%)$ \\
\hline \multicolumn{4}{|l|}{ Surgical procedure } \\
\hline Total or near-total thyroid resection (TR) & $63(38)$ & $30(37)$ & $33(40)$ \\
\hline Partial thyroid resection (PR) & $103(62)$ & $53(64)$ & $50(60)$ \\
\hline Subtotal unilateral thyroid resection & $8(5)$ & $6(7)$ & $2(2)$ \\
\hline Bilateral incomplete lobectomy & $8(5)$ & $7(8)$ & $1(1)$ \\
\hline Hemithyroidectomy (lobectomy) & $32(19)$ & $14(17)$ & $18(21)$ \\
\hline Subtotal thyroidectomy & $55(33)$ & $26(31)$ & $29(35)$ \\
\hline Ipsilateral partial lymphadenectomy & $25(15)$ & $15(18)$ & $10(12)$ \\
\hline \multicolumn{4}{|l|}{ Postoperative treatment } \\
\hline${ }^{131}$ I radio-iodine (RIA) & $106(64)$ & $52(63)$ & $54(65)$ \\
\hline EBR & $11(7)$ & $2(3)$ & $9(11)$ \\
\hline RIA + EBR & $23(14)$ & $11(13)$ & $12(15)$ \\
\hline
\end{tabular}

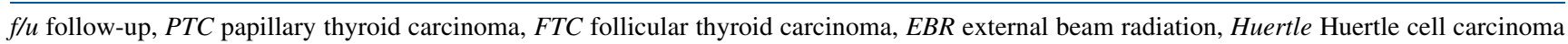


diagnosis was 54.2 years (range 20.2-86). The number of women was more than double that of men (115 vs. 51). Of patients with PTC, 50\% presented with T1 tumors (tumor $<2 \mathrm{~cm}$ ), $23 \%$ with T2 tumors $(>2 \mathrm{~cm}$ and $\leq 4 \mathrm{~cm}$ ), and $27 \%$ with $\mathrm{T} 3$ tumors $(>4 \mathrm{~cm}$ but limited to the thyroid). Because 49 patients $(59 \%)$ with PTC were $<45$ years of age, $73 \%(n=61)$ of all PTC were considered UICC stage I $(<45$ years or $>45$ years and T1N0M0). For patients with FTC, the percentages with $\mathrm{T} 1, \mathrm{~T} 2$, and $\mathrm{T} 3$ tumors were 9,16 , and $75 \%$, respectively. Thirteen $(16 \%)$ patients with FTC had UICC stage I ( $<45$ years: $n=12)$. However, because lymphadenectomy was only sporadically performed (PTC $18 \%$ and FTC 12\%), one cannot exclude the possibility of understaging in UICC stages I and II because of missed positive lymph nodes in patients older than 45 years with tumor sizes T1 or T2 (PTC, $n=6$; FTC, $n=11$ ). For the 6 patients with PTC we used a sensitivity analysis; by assuming stage III disease (positive lymph nodes) no influence on prognostic factors was detected.

\section{Treatment of Well-Differentiated Thyroid Carcinoma}

Total or near-total thyroidectomy (TR) was the surgical procedure of choice in about one-third $(38 \%)$ of the patients (PTC, 37\%; FTC, 40\%) (Table 1). If no total or near-total resection of the thyroid was performed, subtotal thyroidectomy was the most common alternative surgical procedure. Less frequently, hemithyroidectomy (lobectomy) was performed (19\%). Unilateral or bilateral subtotal thyroid resection (unilateral or bilateral incomplete lobectomy) were rarely performed ( $5 \%$ each). During the period of interest (1972-1990), extensive (cervico-central and

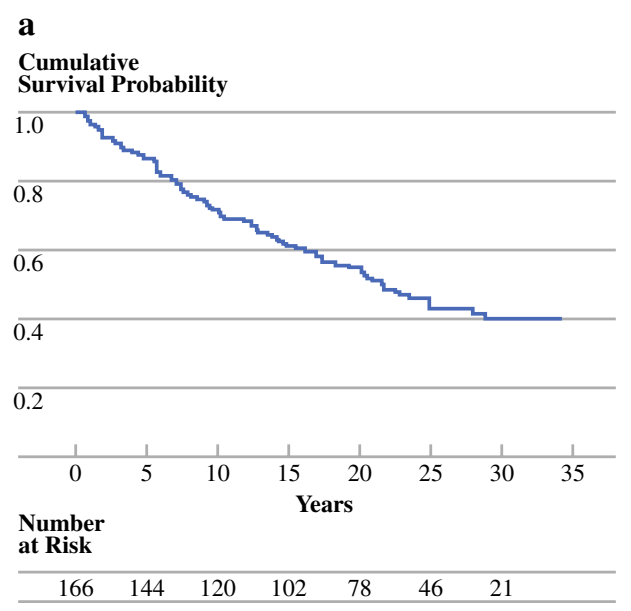

FIG. 1 Kaplan-Meier curves for survival estimates with the number of patients at risk. a Overall survival for patients with complete resection of localized well-differentiated thyroid carcinoma. Median OS was 21.7 (17.1-28.0) years and 20-year survival was $54.9 \%$ (47$63 \%$ ). b Disease-specific survival (DSS) for patients with complete cervico-lateral) lymphadenectomy was only performed in cases highly suspicious of lymph node involvement and was not routinely performed at the University Hospital of Bern. In our cohort, we excluded patients with macroscopic lymph node involvement to avoid the bias of additional extensive lymphadenectomy. Limited lymphadenectomy on the ipsilateral side, removal of a few isolated lymph nodes (berry picking) was reported in 25 patients $(15 \%)$ : $n=15$ in PTC (T2: $n=9$, T3: $n=6) ; n=10$ in FTC (T2: $n=2$, T3: $n=8$ ).

Postoperative treatment consisted of either ${ }^{131} \mathrm{I}$ RIA $(64 \%)$ or external-beam radiation therapy (EBR) $(7 \%)$ or a combination of both radiotherapies (14\%). There were 18 PTC patients and 8 FTC patients who did not receive postoperative treatment (Table 1).

\section{Overall Survival}

A total of 75 patients survived the observation period (45\%). The median OS for all patients was 21.7 years $(95 \%$ confidence interval [95\% CI], 17.1-28) (Fig. 1a and Table 2). The 10- and 20-year OS rates for the patients after complete resection of WDTC were 71 and $55 \%$. The median OS for FTC was 14.2 years, whereas $68 \%$ of patients with PTC (95\%CI, 58-79) survived the observation period (median not reached).

Patients younger than 45 years at the time of resection showed a $97 \%$ (95\% CI, 92-100) and 93\% (95\% CI, 86100) 10 and 20 year OS, respectively. The survival rate after 10 and 20 years in patients aged 45-60 years was $79 \%$ (95\%CI, 66-93) and 46\% (95\% CI, 34-57), respectively, and for patients older than 60 years of age was $58 \%$ (95\% CI, 41-75) and 20\% (95\% CI, 11-30), respectively

b

Cumulative

Survival Probability

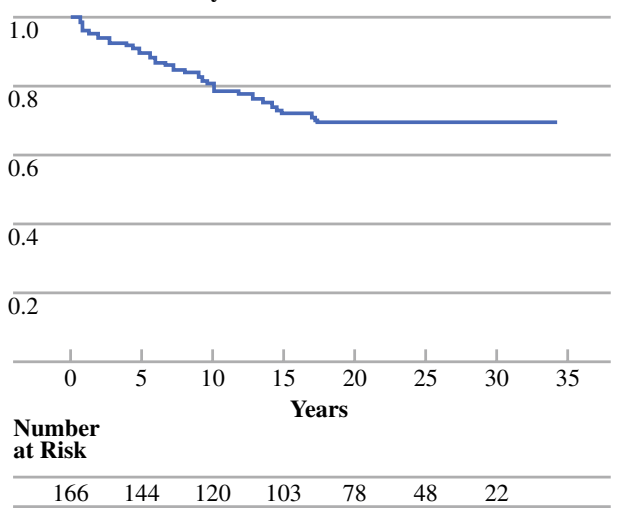

resection of localized well-differentiated thyroid carcinoma. At time of last follow-up, 7 patients were alive with the disease. DSS 20-year survival was $69 \%(62-77 \%)$. The median time to death from disease was $6.7(0.64-17.4)$ years 


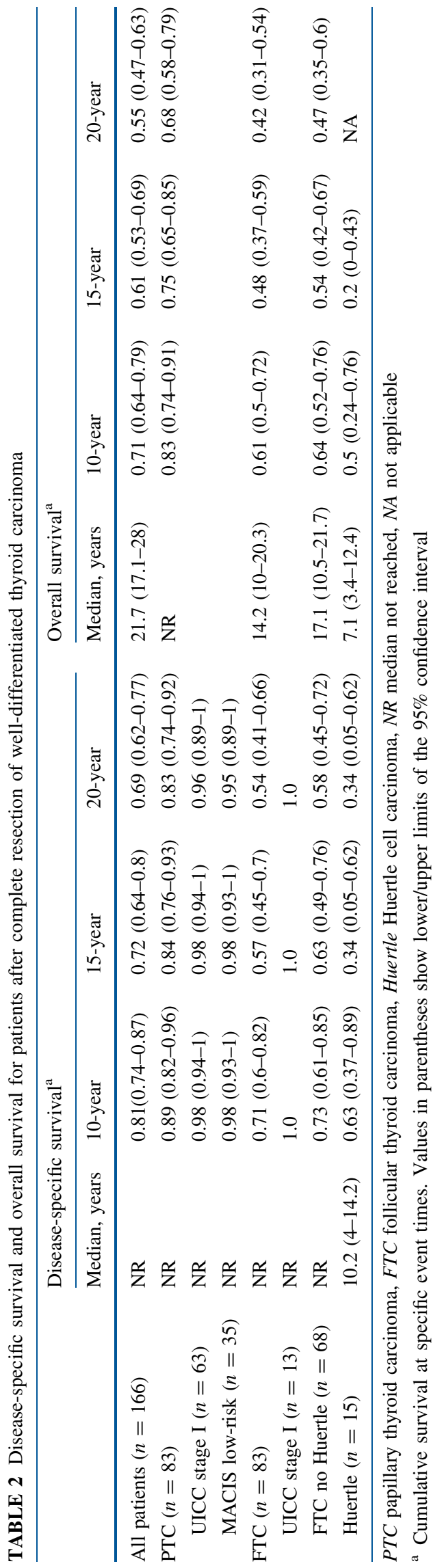

Significant factors associated with worse OS in univariate analysis were older age (hazard ratio [HR] 6.7 for age $>60$ years); male gender (HR 2.1), histology of FTC (HR 2.5), tumor size $>4 \mathrm{~cm}$ (UICC T3) (HR 3.4), no postoperative treatment with ${ }^{131}$ I- RIA (HR 2.3), and treatment with EBR (HR 2.3) (Table 3).

In a multivariate analysis of OS that included age $(<60$ vs. $>60$ years), gender, tumor size ( $\mathrm{T}<3$ vs. T3), histology (PTC vs. FTC), extent of resection (TR vs. PR), postoperative RIA, and postoperative EBR, age was the dominant risk factor (risk ratio $=0.09,95 \% \mathrm{CI}, 0.04-0.20$, $P<.001)$. The only other statistically significant factor for OS was female gender (risk ratio $=0.53,95 \% \mathrm{CI}, 0.30$ $0.95, P=.007)$.

\section{Disease-Specific Survival}

During the observation period, 51 (30.7\%) patients died of WDTC (Fig. 1b). At the time of follow-up, 7 patients where alive with their disease. The DSS for PTC and FTC after 20 years was 83 and 54\%, respectively (Table 2).

The Kaplan-Meier estimates for DSS stratified by age $(P<.001)$, histology $(P<.001)$, and tumor size $(P=.001)$ are shown in Fig. 2a-c. The DSS estimates according to treatment option, extent of resection $(P=$ not significant $[\mathrm{NS}])$, postoperative RIA therapy $(P=.047)$, and EBR $(P=0.002)$ are given in Fig. 3a-c. Univariate analysis showed that patients older than 60 years had 9.2 times higher risk of dying of WDTC than younger patients with this disease (HR 9.17) (Table 3). Other factors that significantly increased the risk of death from WDTC were FTC histology (HR 3.22), tumor size $>4 \mathrm{~cm}$ (HR 4.16), not receiving postoperative RIA therapy (HR 1.92), and receiving EBR (HR 2.69) (Table 3).

In a subgroup analysis of patients with PTC, age $>60$ years $(P<.001)$, tumor size $>4 \mathrm{~cm}(P=.008)$, and receiving EBR therapy $(P=.001)$ were significant factors predicting worse DSS (Table 4). The subgroup analysis for DSS in patients with FTC showed age $>60$ years $(P<.001)$, postoperative RIA therapy $(P=.013)$, and Huertle cell differentiation $(P=.047)$ to be significant factors, whereas tumor size and treatment with EBR lost its statistical significance (Table 4).

In a multivariate analysis of DSS that included age, gender, histology, tumor size $(\mathrm{T}>4 \mathrm{~cm})$, histology (PTC vs. FTC), extent of resection, postoperative RIA treatment, and postoperative EBR, age $>60$ years was the dominant factor (risk ratio [RR] 4.91, 95\%CI 0.029-0.25, $P=.005$ ) with histology being the only other statistically significant factor (RR 2.89 for FTC, $P=.03$ ).

In patients with PTC, the multivariate analysis of significant factors from the univariate analysis (age, tumor size, and receiving EBR), age again significantly 
TABLE 3 Univariate analysis for overall survival and disease-specific survival in 166 patients with resected well-differentiated thyroid cancer

\begin{tabular}{|c|c|c|c|c|c|c|}
\hline \multirow[t]{2}{*}{ Characteristics } & \multicolumn{3}{|c|}{ Disease-specific survival } & \multicolumn{3}{|c|}{ Overall survival } \\
\hline & Hazard ratio & $95 \% \mathrm{CI}$ & $P$ value & Hazard ratio & $95 \% \mathrm{CI}$ & $P$ value \\
\hline Age, $<60$ vs. $\geq 60$ years & 0.11 & $0.06-0.22$ & $<.001^{*}$ & 0.15 & $0.09-0.25$ & $<.001 *$ \\
\hline Gender, male vs. female & 1.57 & $0.79-3.14$ & .16 & 2.09 & $1.20-3.42$ & $<.001 *$ \\
\hline $\begin{array}{l}\text { Histology, PTC vs. FTC } \\
\quad \text { (without Huertle cell ca) }\end{array}$ & $0.31(0.36)$ & $0.17-0.58(0.18-0.73)$ & $<.001 *(.003 *)$ & $0.40(0.45)$ & $0.26-0.62(0.28-0.72)$ & $<.001 *(.003 *)$ \\
\hline $\begin{array}{l}\text { Tumor size, T1, } 2 \text { vs. }>\text { T2 } \\
\quad(<4 \text { vs. } \geq 4 \mathrm{~cm})\end{array}$ & 0.24 & $0.12-0.47$ & $.002 *$ & 0.29 & $0.17-0.50$ & $<.001^{*}$ \\
\hline Radio-iodide therapy, yes vs. no & 0.52 & $0.27-0.99$ & $.047 *$ & 0.43 & $0.25-0.77$ & $<.001 *$ \\
\hline EBR, yes vs. no & 2.69 & $1.13-6.37$ & $.002 *$ & 2.34 & $1.29-4.24$ & $<.001 *$ \\
\hline Resection, PR vs. TR & 1.40 & $0.75-2.63$ & .30 & 1.37 & $0.88-2.10$ & .17 \\
\hline
\end{tabular}

PTC papillary thyroid carcinoma, FTC follicular thyroid carcinoma, EBR external beam radiation, $P R$ partial thyroid resection, TR total or neartotal thyroid resection

HR was calculated with Cox-Mantel, $P$ values calculated with log-rank test

* Statistically significant differences between groups $(P<.05)$

FIG. 2 Kaplan-Meier curves for estimates of disease-specific survival (DSS) for patients stratified by age (a); histology (b); and tumor size (c). a DSS differed significantly between age groups: patients younger than 45 years $(<45$ years $)$, between 45 and 60 years ( $45-60$ years), and older than 60 years ( $>60$ years) $(P<.001)$. b Patients with papillary thyroid carcinoma (PTC) had significantly better DSS than patients with follicular thyroid carcinoma (FTC) stratified into FTC and Huertle cell carcinoma (FTC and Huertle) $(P<.001 ;$ log-rank $)$ c DSS stratified by TNM T-stage (tumor size: T1-T3) were significantly different $(P<.001$; log-rank). Numbers below the graphs show patients at risk at the given time points

\section{a}

Cumulative

Survival Probability

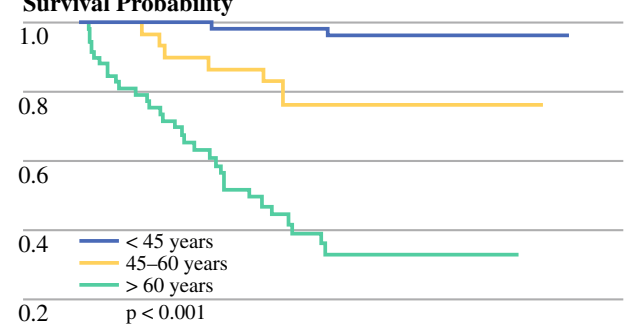

0.2

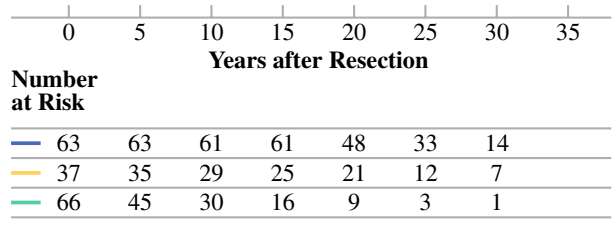

c

Cumulative

Survival Probability

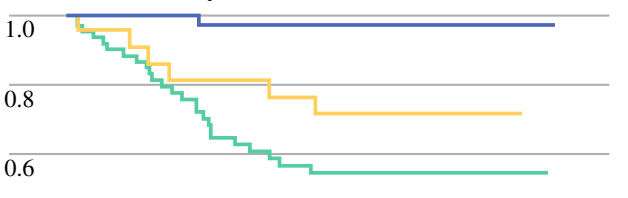

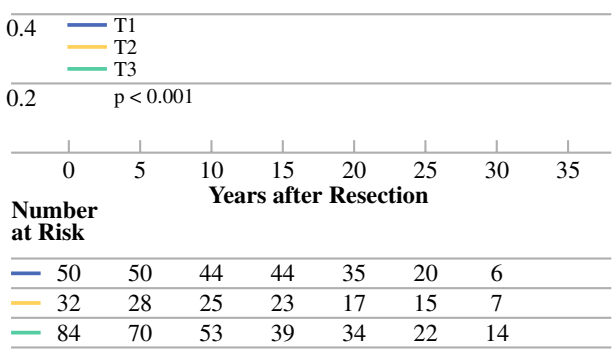

b

Cumulative Survival Probability

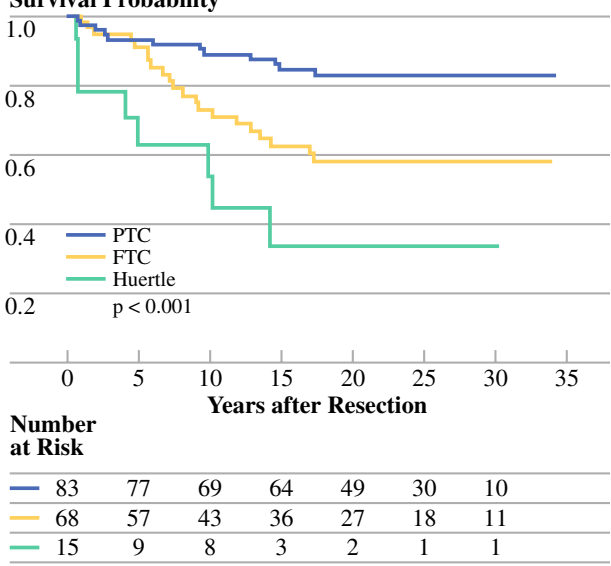

\begin{tabular}{|c|c|c|c|c|c|c|c|}
\hline 0 & 5 & 10 & 15 & 20 & 25 & 30 & 35 \\
\hline \multicolumn{8}{|c|}{ Years after Resection } \\
\hline-83 & 77 & 69 & 64 & 49 & 30 & 10 & \\
\hline$=68$ & 57 & 43 & 36 & 27 & 18 & 11 & \\
\hline-15 & 9 & 8 & 3 & 2 & 1 & 1 & \\
\hline
\end{tabular}


FIG. 3 Kaplan-Meier curves for disease-specific survival (DSS) estimates stratified by extent of thyroid resection (a), by application of postoperative radioiodide treatment (b), and by treatment with postoperative external beam radiation (EBR) (c). a DSS did not differ significantly between patients treated with total/near-total thyroidectomy (total resection, TR) and with partial thyroidectomy (partial resection, PR) $(P=.3$; log-rank $)$. b Patients assigned to and receiving radioiodide treatment after TR of their thyroid cancer (yes) fared better than patients without radio-iodide therapy (no) $(P=.047$; log-rank). c DSS of patients treated with external beam radiation (EBR) after resection (yes) was worse than their counterparts not receiving EBR (no) $(P<.001$; log-rank) (c). Numbers below the graphs show patients at risk at the given time points a

Cumulative

Survival Probability

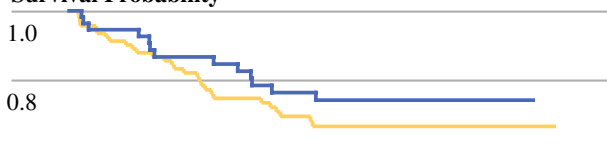

0.6

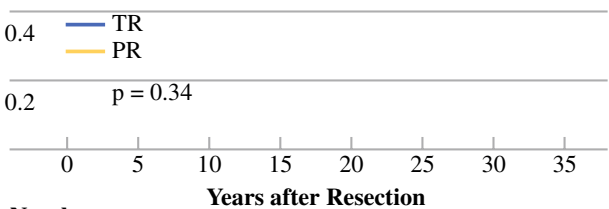

Number

at Risk

\begin{tabular}{lllllll}
\hline-63 & 55 & 48 & 42 & 38 & 31 & 13 \\
\hline 103 & 88 & 72 & 60 & 41 & 17 & 9
\end{tabular}

c

Cumulative

Survival Probability

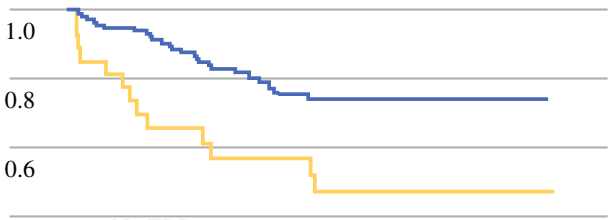

$0.4 \quad$ No EBR

$0.2 \quad \mathrm{p}=0.002$

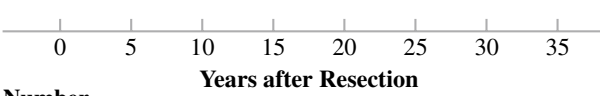

Number

at Risk

\begin{tabular}{lcccccc}
\hline-132 & 120 & 102 & 87 & 68 & 42 & 20 \\
\hline 34 & 23 & 18 & 15 & 10 & 6 & 1
\end{tabular} b

Cumulative

Survival Probability

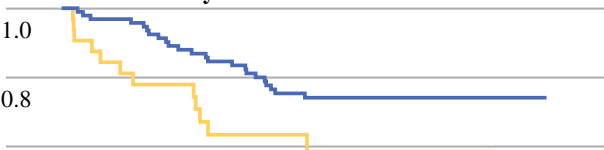

0.6

\begin{tabular}{ll}
\hline $0.4 \quad$ RIA \\
& No RIA \\
\hline 0.2 & $\mathrm{p}=0.047$
\end{tabular}

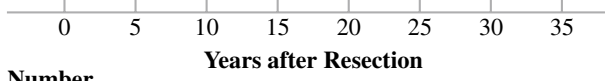

Number
at Risk

\begin{tabular}{lllllll}
\hline-106 & 98 & 86 & 73 & 57 & 40 & 19 \\
\hline-37 & 28 & 21 & 17 & 13 & 2 & 1
\end{tabular}

TABLE 4 Univariate analysis PTC and FTC for disease-specific survival after resection

\begin{tabular}{|c|c|c|c|c|c|c|}
\hline \multirow[t]{2}{*}{ Characteristics } & \multicolumn{3}{|l|}{ PTC } & \multicolumn{3}{|l|}{ FTC } \\
\hline & Hazard ratio & $95 \% \mathrm{CI}$ & $P$ value & Hazard ratio & $95 \% \mathrm{CI}$ & $P$ value \\
\hline Age, $<60$ vs. $>60$ years & 0.06 & $0.01-0.3$ & $<.001 *$ & 0.2 & $0.1-0.42$ & $<.001 *$ \\
\hline Tumor size, T1, 2 vs. $>$ T2 $(<4$ vs. $\geq 4 \mathrm{~cm})$ & 0.15 & $0.03-0.79$ & $.008^{*}$ & 0.85 & $0.33-2.17$ & .73 \\
\hline Radio-iodide therapy, yes vs. no & 0.52 & $0.13-2.11$ & .28 & 0.39 & $0.19-0.80$ & $.013 *$ \\
\hline EBR, yes vs. no & 5.29 & $0.92-30.33$ & $.001 *$ & 1.63 & $0.65-4.14$ & .23 \\
\hline Resection, PR vs. TR & 1.22 & $0.38-3.91$ & .75 & 1.59 & $0.76-3.33$ & .24 \\
\hline
\end{tabular}

$P T C$ papillary thyroid carcinoma, FTC follicular thyroid carcinoma, EBR external beam radiation, $P R$ partial thyroid resection, $T R$ total or neartotal thyroid resection

HR was calculated with Cox-Mantel, $P$-values calculated with log-rank test

*Statistically significant differences between groups $(P<.05)$

\section{Extent of Resection}

WDTC was most often removed by partial resection of the thyroid gland (PR). Subtotal unilateral or bilateral thyroid resection (10\%) or hemithyroidectomy (lobectomy) (19\%) had the goal of complete resection of the cancer alone, avoiding potential morbidity of more extensive resection. The scope of subtotal thyroidectomy was primarily the removal of the tumor with as much thyroid tissue as possible to minimize the risk of unapparent synchronous carcinoma and to allow effective postoperative RIA therapy (subtotal thyroidectomy, 33\%) (Table 1). Total or near-total (less than 
$1 \mathrm{~g}$ thyroid tissue left) thyroidectomy has the advantage that synchronous tumors and tissue for metachronous disease is removed (total thyroidectomy, 38\%). Additional advantages of total thyroidectomy is the increased efficiency of postoperative RIA therapy and the decreased risk of damage to the recurrent laryngeal nerve in case a second surgical intervention is necessary as a result of recurrent disease.

Table 5 shows the DSS rate after partial thyroidectomy and after total removal of the gland. For all patients, the 10- and 20-year DSS after partial thyroidectomy was 77 and $66 \%$, respectively, and the 10- and 20-year DDS after total thyroidectomy was 87 and $74 \%$, respectively. The Kaplan-Meier DSS estimates, stratified by histology and the extent of resection, are shown in Fig. 4a-c; DSS after PR and TR for PTC was 82 and $84 \%$, respectively $(P=\mathrm{NS})$ (Fig. 4a); DSS rates for FTC were 47 and $63 \%$ after $\mathrm{PR}$ and $\mathrm{TR}$, respectively $(P=\mathrm{NS})$ (Fig. $4 \mathrm{~b})$. No statistically significant difference in DSS for the extent of resection could be detected in patients with FTC, even when the more aggressive Huertle cell carcinoma was excluded ( $P=\mathrm{NS}$ ) (Fig. 4c). Hence, in this group of selected patients who had complete removal of their WDTC confined to the thyroid gland, with no macroscopically apparent lymph node invasion or distant metastases, the extent of resection of the surrounding thyroid tissue did not influence DSS. In this context, it is important to note that lymphadenectomy was not routinely performed, and if done at the surgeon's discretion, was limited to ipsilateral incomplete lymphadenectomy.

Postoperative complications were noted in 51 patients. Postoperative transient recurrent paresis was detected with postoperative laryngoscopy in 31 patients, and 1 patient required short-term tracheostomy due to bilateral nerve paresis (10.6\% with 292 nerves at risk); 17 of these nerve lesions occurred in patients treated with PR and 14 in patients with TR of the thyroid gland ( $P=0.42$, Fisher exact test). Transient hypocalcaemia was noted in 22 patients (13\%) and occurred significantly more often in patients who underwent total or near-total thyroid resection $(P=.001$, Fisher exact). One patient each had bleeding or infection, respectively, that required intervention.

\section{DISCUSSION}

\section{Findings}

In this analysis of 166 patients with completely resected, well-differentiated carcinoma that did not extend beyond the thyroid capsule, and who were treated at a tertiary care center over an 18-year period, the DSS of PTC and FTC were 83 and 54\%, respectively, during a median follow-up

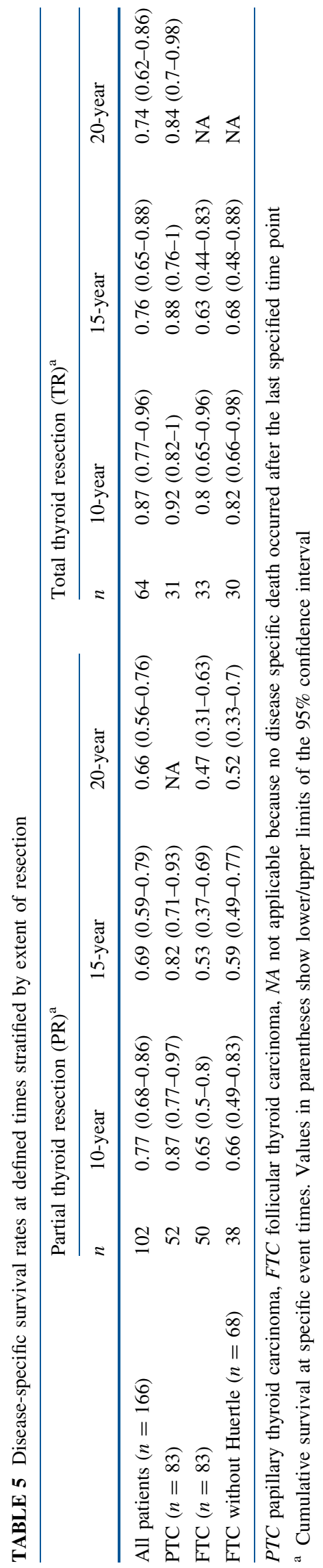


FIG. 4 Kaplan-Meier curves for disease-specific survival (DSS) estimates stratified by extent of resection in papillary thyroid carcinoma (PTC) (a), follicular thyroid carcinoma (FTC) (b), and FTC excluding Huertle cell carcinoma (c). a DSS in patients with complete resection of PTC was independent on the extent of thyroid removal (TR: total thyroid resection, $\mathrm{PR}$ : partial thyroid resection) $(P=.75)$. b DSS in patients with complete resection of follicular thyroid carcinoma (FTC) was not significantly different between TR and PR $(P=.37$; log-rank $)$ and remained below significance levels when patients with Huertle cell carcinoma were excluded $(P=.65 ; \log$ rank $)(\mathbf{c})$

\section{a}

\section{Cumulative}

Cumulative
Survival Probability
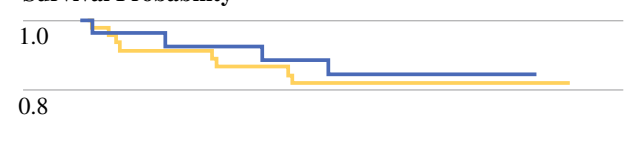

0.6

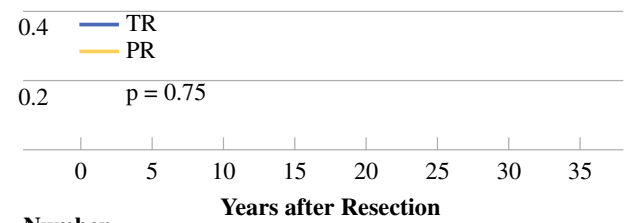

Number

at Risk

\begin{tabular}{|c|c|c|c|c|c|c|}
\hline-31 & 30 & 26 & 25 & 22 & 20 & 7 \\
\hline-52 & 47 & 43 & 38 & 27 & 10 & 3 \\
\hline
\end{tabular}

b

Cumulative

Survival Probability

1.0

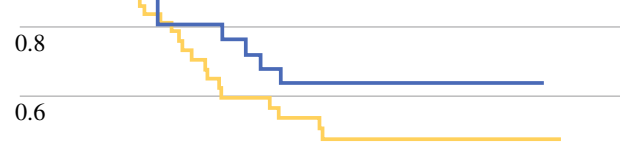

\begin{tabular}{l}
\hline $0.4-$ TR \\
- PR
\end{tabular}

$0.2 \quad \mathrm{p}=0.24$

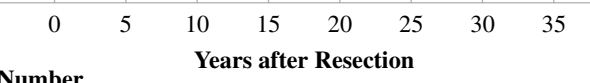

Number

\begin{tabular}{lllllll}
\multicolumn{1}{l}{ at Risk } \\
\hline-33 & 26 & 22 & 18 & 17 & 12 & 6 \\
\hline 50 & 40 & 28 & 21 & 13 & 7 & 6
\end{tabular}

c

Cumulative

Survival Probability

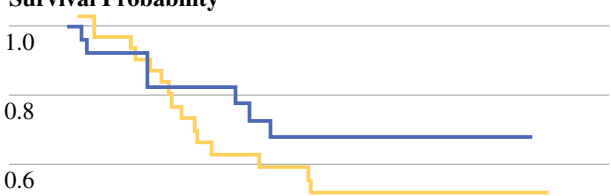

0.6

$0.4=\mathrm{TR}$
$=\mathrm{PR}$

$0.2 \quad p=0.34$

\begin{tabular}{|c|c|c|c|c|c|c|c|}
\hline$\perp$ & $\perp$ & $\perp$ & $\perp$ & 1 & & $\perp$ & 1 \\
\hline 0 & 5 & 10 & 15 & 20 & 25 & 30 & 35 \\
\hline \multicolumn{8}{|c|}{ Years after Resection } \\
\hline-30 & 24 & 20 & 17 & 16 & 12 & 6 & \\
\hline-38 & 33 & 23 & 19 & 12 & 6 & 5 & \\
\hline
\end{tabular}

of 27 years. The last death resulting from thyroid cancer occurred 18 years after the initial resection. The established prognostic factors for WDTC of age, gender, histology, tumor size, and postoperative RIA therapy, were significant factors for DSS in the univariate analysis. Age was the dominant risk factor in a multivariate analysis. Application of EBR after resection of the thyroid cancer resulted in significantly decreased DSS. The extent of thyroid resection, in addition to the complete removal of the carcinoma, did not influence DSS.

\section{Survival}

Long-term follow-up studies from the United States and Asia describe 20-year DSS rates for PTC without distant metastasis of $92-96 \%$ (99\%). ${ }^{7,36,37}$ These survival rates are about $10 \%$ higher than in our population. This discrepancy could be the result of etiologic differences that correlate with geography, as reported 10-year and 20-year DSS from Sweden, Germany, and Austria, where goiter is endemic or prevented by iodide substitution, were similar or worse compared with this study. ${ }^{3,9,38,39}$ Whereas tumor size (median $50 \mathrm{~mm}$ ) was slightly higher, the age distribution, which was a major risk factor, was similar in our cohort (median age, 41.3 years in the PTC group) with those reported from countries without endemic goiter (United States, Asia). Studies from regions with endemic goiter, show that the proportion of FTC in patients with WDTC is higher in these regions. Lerch et al. from Germany reported 40\% FTC; Passler et al., 33\%; and Steinmüller et al., $30 \% .^{39-41}$ In our population, FTC made up half of the WDTC. However, the less favorable DSS in WDTC from Europe could not be explained by the higher rate of FTCs because the DSS for each histologic type was lower than in analysis from outside Europe.

Considering the long-term risk of death from completely resected WDTC confined to the thyroid gland found in this study (17\% for PTC and $46 \%$ for FTC), the axiom that this disease displays a benign course might not be entirely appropriate, and treatment nihilism is surely inadequate. 
This is especially true for older patients, where the tendency for "watchful waiting" in cases of newly detected thyroid lesions is misguided. In our analysis, only a third of patients older than 60 years of age did not die of their disease. The fact that people grow to older ages and that the incidence of WDTC peaks in the sixth decade adds further weight to the importance of correct initial treatment. ${ }^{3}$

An important finding of this study is that no patients died of their disease more than 18 years after complete resection of the thyroid cancer. Although this is likely the result of the very good prognosis for young patients and the risk of death from cancer independent causes (competing causes) in the older population, it is noteworthy. Analyzing the Kaplan-Meier survival curves of other long-term studies, it becomes evident that, indeed, death resulting from WDTC is unlikely more than 15 years after resection. ${ }^{3,7,36,37,39-41}$ This finding has direct implications for further studies evaluating risk factors and prognostic parameters. Follow-up periods of 10 to 15 years are sufficient for a clinically significant evaluation of WDTC.

\section{Prognostic Parameters}

Major prognostic factors for disease recurrence and DSS in WDTC, namely patient age, gender, histology, tumor size, presence of distant metastasis, extension beyond the thyroid capsule, and completeness of resection have been identified mostly based on large populationbased studies (cancer registries) with mid- to long-term follow-ups. Because of the restrictions we used in our attempt to limit our analysis to a more homogeneous patient population, some of these factors could not be evaluated (notably, lymph node metastasis). The findings in this study confirm the reported prognostic parameters in univariate analysis. "Age" was the all-dominant factor for DSS followed by "histology" in the multivariate analysis. However, the study likely lacked the power to allow conclusive analysis of multiple parameters. Here we also analyzed two controversial prognostic parameters; extent of thyroid resection and postoperative application of EBR.

\section{Extent of Resection}

The results presented here are from well-documented interventions on primary tumors confined to the thyroid gland, with no macroscopic spread to adjacent lymph nodes. The surgical approach during the observation period infrequently extended to regional lymph node sampling $(14 \%)$. This is why we decided not to take lymphadenectomy into account, because lymphadenectomy similar to current standards could not be verified. TR of the thyroid tissue did not improve DSS compared with PR. Although the Kaplan-Meier survival curves trended toward better outcomes after TR, this effect was much less important than other risk factors, namely, postoperative RIA therapy. Most importantly, in patients with PTC, the WDTC variant that is often multifocal and bilateral, there was no benefit for TR, and the survival curves for TR and PR almost overlap.

These results agree with most of the analysis from large single-institution and SEER database mid-term studies that favor hemithyroidectomy (lobectomy) of the affected side over total thyroidectomy. ${ }^{14,19,20,24-29,31}$ Indeed, recommendations and consensus guidelines advocating total or near-total thyroidectomy in high-risk PTCs, but not necessarily in single, small, low-risk, intrathyroidal lesions, are not based on evidence demonstrating improved DSS, but rather on an understanding of the disease being multifocal with an increased risk of recurrence and the option to treat small residual thyroid cancer with RIA more effectively. ${ }^{42,43}$ In addition, proponents of limited resection base their strategy on publications reporting increased postsurgical complications with more extensive resection. ${ }^{23}$ In the current analysis, we found an increased rate of transient postoperative hypocalcemia in patients treated with TR, but no difference in the rate of transient nerve paralysis. Previous studies on TR at our institution could not rectify limited resections based on nerve injury rates. ${ }^{44}$ However, guidelines recommending TR as the treatment of choice also take into consideration that secondary total thyroidectomy for disease recurrence has a higher risk of complications than primary total thyroidectomy. Very recently, Bilimoria et al. advocated TR in all PTC patients with tumors greater than $1 \mathrm{~cm}$ based on a very large population from the National Cancer Data Base. ${ }^{45}$ However, the limitations of this kind of study do not permit optimal treatment recommendations or suggestions (see comments by Shah) ${ }^{46}$ Most importantly, performance of lymphadenectomy was not taken into account in Bilimoria's analysis and could well explain the improved survival after complete resection of the thyroid gland.

Generally, it must be assumed that retrospective analyses of the extent of resection based on surgical reports overestimate the rate of true total thyroidectomies. Schwab et al. found residual thyroid tissue $>1 \mathrm{~mL}$ in more than one-third of patients reportedly treated with "total thyroidectomy" in postoperative scintigraphies (uptake measures). ${ }^{38}$ The amount of thyroid tissue left behind would not fulfill the criteria of "near-total thyroidectomy"; however, the volume of the remnant was below the limit that could compromise RIA. We cannot exclude that this reporting error could explain why there was no difference in DSS after PR compared with total thyroidectomy. 


\section{External Beam Radiation Therapy (EBR)}

Our results show a negative effect of postoperative EBR on DSS. This may be the result of patient selection, for example, those with poor prognostic parameters who required EBR, either combined with RIA or alone. However, age, histology, tumor size, surgery performed, and completeness of resection of the 34 patients who received EBR were similar to the group who did not receive EBR. Because we included only patients who had complete tumor resections, the negative effect of EBR in this select group of patients with low to mid risk tumors is likely to be real. However, one must consider the potentially suboptimal 30 Gy doses applied compared with the 60-70 Gy used more recently. ${ }^{47,48}$ Lower doses were used historically because older radiotherapy methods could not deliver an adequate dose of radiation to the thyroid bed without exceeding the tolerance of the posteriorly located spinal cord. In 2003, Ford et al. reported that local recurrence and OS are related to the dose prescribed, based on a small cohort $(n=41)$, suggesting a dose of a least 50 Gy is needed to impact local control and possibly survival. ${ }^{49}$ Our findings concur with the recent management guidelines put forth by the American Thyroid Association Guidelines Taskforce, which considers EBR only for patients with gross residual tumors and in whom further surgery or RIA treatment are likely to be ineffective. ${ }^{42}$ According to the relative radioresistance and slow regression rate of PTC, Tuttle et al. reported no apparent advantage of EBR for OS, but significant morbidities. ${ }^{50}$ Our findings strongly support this notion for patients who have limited disease and complete tumor resection.

\section{Limitations of This Study}

The most important limitation of this study is the small number of patients included. The inherent risk that significant differences could not be detected may be crucial in assessing the influence of the extent of thyroid resection. Indeed, with 166 patients included and a 20-year DSS rate of $66 \%$ in patients with partially resected thyroid, the DSS rate for TR would have to vary by $>20 \%$ in order to become statistically significant (we found an $8 \%$ difference in DSS). However, the limited power of this study is partly compensated for by the very long follow-up, which allowed us to estimate trends in the Kaplan-Meier survival curves. Whereas a certain divergence of the curves depicting TR and PR in FTC over a 30-year time span was observed, this was not true in the DSS curves of patients with resected PTC. Here, the almost overlapping curves would suggest that the nonsignificant difference between TR and PR is real, and not merely the result of the study being underpowered. Because most recognized prognostic factors reach statistical significance despite the rather small number of patients observed also suggests that the extent of thyroid resection without lymphadenectomy is, at most, a minor factor for DSS.

Accordingly, the effect of RIA after incomplete resection could not be estimated because of a lack of sufficient power (only 37 patients did not receive RAI, and of these, 11 were treated with EBR). Moreover, of these 26 patients, only 3 were treated with total thyroidectomy and none died of thyroid cancer.

Another limitation is the exclusion of lymph node metastases as prognostic factors. Unlike other studies, we decided not to report or include nodal involvement because lymphadenectomy during the period in question was heterogeneous and dependent on the individual physician's decision. The quality of lymphadenectomy cannot be evaluated retrospectively. Adding lymph node metastases to the survival analysis dating back more than 15 years runs the risk of coming to the wrong conclusion. Scintigraphic uptake and pathologic analysis of retrospective studies by Schwab et al. showed that $56 \%$ of lymph node dissections did not fulfill the quality standards, even when ipsilateral and centrocervical lymphadenectomy is reported by the surgeon. ${ }^{38}$ Rather than use uncertain data, we preferred to exclude this factor from the analysis. Although lymph node involvement is not an independent risk factor, with the exclusion of lymphadenectomy, this study cannot deny the possibility that complete thyroidectomy combined with appropriate lymphadenectomy might improve DSS.

\section{Strength of This Study}

Studies with very long-term follow-up are often hampered by inconsistencies in the applied treatment over time. The major advantages of the study are the very-long follow-up and the well-defined group of WDTC patients. Unlike most other studies reporting 20-year survival rates, this single-institutional database with detailed, prospectively collected information was limited to a period during which treatment standards and reporting remained the same. It is likely that patients in single-institutional studies reporting data over a 40-year period were treated differently, even when the surgical approaches were equally termed. Technical improvements, exposure of the recurrent laryngeal nerve, the definition of "subtotal," "near total," "total thyroidectomy," and most importantly, of complete lymphadenectomy has changed over the last 4 decades. ${ }^{37}$ An analysis of these studies for independent factors runs the risk of a time and treatment bias. In addition, large observational studies based on population-based databases suffer from a lack of detailed descriptions of the tumor characteristics and treatment strategies. This is where the strength and value of this study lies; in the long-term 
analysis of a small, very well defined group of WDTC patients treated in a homogeneous manner.

\section{Conclusions}

Our findings agree with most of the literature reporting on prognostic factors in WDTC. The unusually long followup of a median 27 years showed that 18 years after a complete resection, no more patients died of thyroid cancer, but that disease-specific death rates of WDTC in Europe are by no means negligible. Whereas EBR (at doses lower than the present day standard) after complete resection of WDTC confined to the thyroid negatively affected DSS, the effect of extensive thyroid resection (without additional lymphadenectomy) compared with partial resection on DSS was, at most, minimal (especially in PTC, where the survival curves overlapped). Future studies should focus on the value of total or near-total thyroidectomy combined with complete (ipsilateral and cervico-central) lymphadenectomy compared with unilateral thyroidectomy with/without ipsilateral lymphadenectomy. Our data, furthermore, suggest that for future studies an observation period of 15 years is probably sufficient. In addition, determination of any molecular differences in PTC among Europeans, Asians, and Americans could help explain the significant difference in DSS of PTC reported by others and confirmed by our findings.

\section{REFERENCES}

1. Schlumberger MJ. Papillary and follicular thyroid carcinoma. N Engl J Med. 1998;338:297-306.

2. Sherman SI. Thyroid carcinoma. Lancet. 2003;361:501-11.

3. Lundgren CI, Hall P, Ekbom A, Frisell J, Zedenius J, Dickman PW. Incidence and survival of Swedish patients with differentiated thyroid cancer. Int J Cancer. 2003;106:569-73.

4. Gilliland FD, Hunt WC, Morris DM, Key CR. Prognostic factors for thyroid carcinoma. A population-based study of 15,698 cases from the Surveillance, Epidemiology and End Results (SEER) program 1973-1991. Cancer. 1997;79:564-73.

5. Lundgren CI, Hall P, Dickman PW, Zedenius J. Clinically significant prognostic factors for differentiated thyroid carcinoma: a population-based, nested case-control study. Cancer. 2006;106: 524-31.

6. Lang BH, Lo CY, Chan WF, Lam AK, Wan KY. Classical and follicular variant of papillary thyroid carcinoma: a comparative study on clinicopathologic features and long-term outcome. World J Surg. 2006;30:752-8.

7. Hay ID, Thompson GB, Grant CS, Bergstralh EJ, Dvorak CE, Gorman CA, et al. Papillary thyroid carcinoma managed at the Mayo Clinic during six decades (1940-1999): temporal trends in initial therapy and long-term outcome in 2444 consecutively treated patients. World J Surg. 2002;26:879-85.

8. Lang B, Lo CY, Chan WF, Lam KY, Wan KY. Restaging of differentiated thyroid carcinoma by the sixth edition AJCC/UICC TNM staging system: stage migration and predictability. Ann Surg Oncol. 2007;14:1551-9.
9. Passler C, Prager G, Scheuba C, Kaserer K, Zettinig G, Niederle B. Application of staging systems for differentiated thyroid carcinoma in an endemic goiter region with iodine substitution. Ann Surg. 2003;237:227-34.

10. Hay ID, Klee GG. Thyroid cancer diagnosis and management. Clin Lab Med. 1993;13:725-34.

11. Blankenship DR, Chin E, Terris DJ. Contemporary management of thyroid cancer. Am J Otolaryngol. 2005;26:249-60.

12. Mazzaferri EL. Long-term outcome of patients with differentiated thyroid carcinoma: effect of therapy. Endocr Pract. 2000;6:469-76.

13. D'Avanzo A, Treseler P, Ituarte PH, Wong M, Streja L, Greenspan FS, et al. Follicular thyroid carcinoma: histology and prognosis. Cancer. 2004;100:1123-9.

14. McConahey WM, Hay ID, Woolner LB, van Heerden JA, Taylor WF. Papillary thyroid cancer treated at the Mayo Clinic, 1946 through 1970: initial manifestations, pathologic findings, therapy, and outcome. Mayo Clin Proc. 1986;61:978-96.

15. Cady B, Sedgwick CE, Meissner WA, Bookwalter JR, Romagosa V, Werber J. Changing clinical, pathologic, therapeutic, and survival patterns in differentiated thyroid carcinoma. Ann Surg. 1976;184:541-53.

16. Farrar WB, Cooperman M, James AG. Surgical management of papillary and follicular carcinoma of the thyroid. Ann Surg. 1980;192:701-4.

17. Starnes HF, Brooks DC, Pinkus GS, Brooks JR. Surgery for thyroid carcinoma. Cancer. 1985;55:1376-81.

18. Clark OH, Levin K, Zeng QH, Greenspan FS, Siperstein A. Thyroid cancer: the case for total thyroidectomy. Eur J Cancer Clin Oncol. 1988;24:305-13.

19. Gemsenjager E, Heitz PU, Martina B. Selective treatment of differentiated thyroid carcinoma. World J Surg. 1997;21:546-51; discussion 51-2.

20. Shah JP, Loree TR, Dharker D, Strong EW, Begg C, Vlamis V. Prognostic factors in differentiated carcinoma of the thyroid gland. Am J Surg. 1992;164:658-61.

21. Harness JK, Fung L, Thompson NW, Burney RE, McLeod MK. Total thyroidectomy: complications and technique. World J Surg. 1986;10:781-6.

22. Bilimoria KY, Bentrem DJ, Ko CY, Stewart AK, Winchester DP, Talamonti MS, et al. Extent of surgery affects survival for papillary thyroid cancer. Ann Surg. 2007;246:375-81; discussion 81-4.

23. Schroder DM, Chambors A, France CJ. Operative strategy for thyroid cancer. Is total thyroidectomy worth the price? Cancer. 1986;58:2320-8.

24. Kebebew E, Clark OH. Differentiated thyroid cancer: "complete" rational approach. World J Surg. 2000;24:942-51.

25. Udelsman R, Shaha AR. Is total thyroidectomy the best possible surgical management for well-differentiated thyroid cancer? Lancet Oncol. 2005;6:529-31.

26. Haigh PI, Urbach DR, Rotstein LE. AMES prognostic index and extent of thyroidectomy for well-differentiated thyroid cancer in the United States. Surgery. 2004;136:609-16.

27. Wanebo H, Coburn M, Teates D, Cole B. Total thyroidectomy does not enhance disease control or survival even in high-risk patients with differentiated thyroid cancer. Ann Surg. 1998;227: 912-21.

28. Jonklaas J, Sarlis NJ, Litofsky D, Ain KB, Bigos ST, Brierley JD, et al. Outcomes of patients with differentiated thyroid carcinoma following initial therapy. Thyroid. 2006;16:1229-42.

29. Haigh PI, Urbach DR, Rotstein LE. Extent of thyroidectomy is not a major determinant of survival in low- or high-risk papillary thyroid cancer. Ann Surg Oncol. 2005;12:81-9.

30. Shaha AR, Shah JP, Loree TR. Low-risk differentiated thyroid cancer: the need for selective treatment. Ann Surg Oncol. 1997;4:328-33. 
31. Taylor T, Specker B, Robbins J, Sperling M, Ho M, Ain K, et al. Outcome after treatment of high-risk papillary and non-Hurthle-cell follicular thyroid carcinoma. Ann Intern Med. 1998;129:622-7.

32. Mazzaferri EL. Papillary and follicular thyroid cancer: selective therapy. Compr Ther. 1981;7:6-14.

33. Greene F. AJCC cancer staging handbook: TNM classification of malignant tumors. 6th ed. New York: Springer/Verlag; 2002.

34. Kaplan E, Meier P. Nonparametric estimation from incomplete observations. J Am Stat Assoc. 1958;1:457-71.

35. Therneau TM GP. Modeling survival data: Extending the Cox model. New York: Springer; 2000.

36. Shaha AR, Shah JP, Loree TR. Risk group stratification and prognostic factors in papillary carcinoma of thyroid. Ann Surg Oncol. 1996;3:534-8.

37. Lang BH, Lo CY, Chan WF, Lam KY, Wan KY. Prognostic factors in papillary and follicular thyroid carcinoma: their implications for cancer staging. Ann Surg Oncol. 2007;14:730-8.

38. Schwab R, Wieler H, Birtel S, Ostwald-Lenz E, Kaiser KP, Becker HP. Confronting the practice of surgery on differentiated thyroid cancer with current guidelines in Germany. A multicenter trial. Nuklearmedizin. 2005;44:185-91.

39. Lerch H, Schober O, Kuwert T, Saur HB. Survival of differentiated thyroid carcinoma studied in 500 patients. J Clin Oncol. 1997; 15:2067-75.

40. Steinmuller T, Klupp J, Rayes N, Ulrich F, Jonas S, Graf KJ, et al. Prognostic factors in patients with differentiated thyroid carcinoma. Eur J Surg. 2000;166:29-33.

41. Passler C, Scheuba C, Prager G, Kaczirek K, Kaserer K, Zettinig $\mathrm{G}$, et al. Prognostic factors of papillary and follicular thyroid cancer: differences in an iodine-replete endemic goiter region. Endocr Relat Cancer. 2004;11:131-9.
42. Cooper DS, Doherty GM, Haugen BR, Kloos RT, Lee SL, Mandel SJ, et al. Management guidelines for patients with thyroid nodules and differentiated thyroid cancer. Thyroid. 2006;16: 109-42.

43. Taskforce TC. AACE/AAES medical/surgical guidelines for clinical practice: management of thyroid carcinoma, 2001.

44. Seiler CA, Vorburger SA, Burgi U, Candinas D, Schmid SW. Extended resection for thyroid disease has less operative morbidity than limited resection. World J Surg. 2007;31:1005-13.

45. Bilimoria KY, Zanocco K, Sturgeon C. Impact of surgical treatment on outcomes for papillary thyroid cancer. Adv Surg. 2008;42:1-12.

46. Shah JP. Re: extent of surgery affects papillary thyroid cancer. Ann Surg. 2008;247:1082-3; author reply 83-4.

47. Rosenbluth BD, Serrano V, Happersett L, Shaha AR, Tuttle RM, Narayana A, et al. Intensity-modulated radiation therapy for the treatment of nonanaplastic thyroid cancer. Int J Radiat Oncol Biol Phys. 2005;63:1419-26.

48. Brierley J, Tsang R, Panzarella T, Bana N. Prognostic factors and the effect of treatment with radioactive iodine and external beam radiation on patients with differentiated thyroid cancer seen at a single institution over 40 years. Clin Endocrinol (Oxf). 2005; 63:418-27.

49. Ford D, Giridharan S, McConkey C, Hartley A, Brammer C, Watkinson JC, et al. External beam radiotherapy in the management of differentiated thyroid cancer. Clin Oncol ( $R$ Coll Radiol). 2003;15:337-41.

50. Tuttle M, Robbins R, Larson SM, Strauss HW. Challenging cases in thyroid cancer: a multidisciplinary approach. Eur J Nucl Med Mol Imaging. 2004;31:605-12. 